\title{
Incidental diagnosis of lung adenocarcinoma following coronavirus OC 43 severe pneumonia
}

\author{
Carlo Iadevaia, ${ }^{1}$ Fabio Perrotta, ${ }^{2}$ Grazia Mazzeo ${ }^{1,3}$ Francesco Saverio Cerqua, ${ }^{1}$ Gennaro Mazzarella, ${ }^{1}$ \\ Salvatore Guarino, ${ }^{4}$ Roberto Parrella, ${ }^{5}$ Andrea Bianco ${ }^{1,3}$ \\ ${ }^{1}$ Department of Translational Medical and Surgical Science, University of Campania Luigi Vanvitelli, Naples; \\ ${ }^{2}$ Department of Medicine and Health Sciences "V. Tiberio", University of Molise, Campobasso; ${ }^{3}$ COVID Unit \\ Vanvitelli, University of Campania Luigi Vanvitelli, Naples; ${ }^{4}$ Department of Radiology, Monaldi Hospital, A.O.R.N. dei \\ Colli, Naples; ${ }^{5}$ Department of Infectious Diseases, COVID Unit Cotugno Hospital, A.O.R.N. dei Colli, Naples, Italy
}

\begin{abstract}
Viral infections are frequent among patients with thoracic malignancies prompted by dysregulation of innate and adaptive immune response. Clinical symptoms and radiological findings of both viral pneumonia and lung adenocarcinoma may overlap resulting in diagnostic and clinical challenges. We present the case of a woman admitted to our department presenting with an acute manifestation of coronavirus OC43 pneumonia with underlying undiagnosed lung adenocarcinoma.
\end{abstract}

Correspondence: Fabio Perrotta, Department of Medicine and Health Sciences "V. Tiberio" - University of Molise, Via F. De Sanctis, 86100 Campobasso, Italy.

E-mail: fabio.perrotta@unimol.it

Key words: Coronavirus OC43; lung adenocarcinoma; NSCLC; viral pneumonia; acute respiratory failure.

Contributions: All the authors made a substantive intellectual contribution. All the authors have read and approved the final version of the manuscript and agreed to be accountable for all aspects of the work.

Conflict of interest: The authors declare that they have no competing interests, and all authors confirm accuracy.

Ethics approval and consent to participate: No ethical committee approval was required for this case report by the Department, because this article does not contain any studies with human participants or animals.

Consent for publication: The patient gave her written consent to use his personal data for the publication of this case report and any accompanying images.

Received for publication: 14 April 2020.

Accepted for publication: 22 June 2020.

${ }^{\circ}$ Copyright: the Author(s), 2020

Licensee PAGEPress, Italy

Monaldi Archives for Chest Disease 2020; 90:1313

doi: 10.4081/monaldi.2020.1313

This article is distributed under the terms of the Creative Commons Attribution Noncommercial License (by-nc 4.0) which permits any noncommercial use, distribution, and reproduction in any medium, provided the original author(s) and source are credited.

\section{Introduction}

Cancer patients are particularly susceptible to develop lung infectious diseases, mainly caused by viruses and bacteria. The recent outbreak of SARS-CoV-2 infection has highlighted that patients with cardiovascular, respiratory and metabolic pre-existing diseases experience poor outcomes once infected [1-4]. Adenocarcinoma is the most common subtype of lung cancer particularly in young women who have never smoked [5]. Clinical presentation is often aspecific and differential diagnosis includes a broad spectrum of nosological entities [6,7]. Computed tomography (CT) scan in invasive adenocarcinoma usually shows heterogeneous patterns including consolidations, ground glass opacities (GGOs) or partly solid hyperdense lesions, single or multifocal. Differential diagnosis between viral pneumonia and malignant disease progression in patients suffering from lung cancer can be very challenging due to the similar radiological characteristics $[8,9]$. We present a case that highlights difficulties in differential diagnosis of a patient with acute lower respiratory viral infection superimposed on lung adenocarcinoma.

\section{Case Report}

In December 2019, a 42-year old female non-smoker was seen in outpatient setting complaining of 10-day history of chest pain, nocturnal fever and mild dyspnea. Past medical history included gastroesophageal reflux, gastritis and uterine myomas. CT scan showed left lower lobe parenchymal consolidation with signs of air bronchogram. The patient was treated with antibiotic therapy including ceftriaxone and ciprofloxacin. Following a transient clinical improvement, after 14 days the patient exhibited relapsed dyspnoea with asthenia and mild cough, mucoid expectoration; peripheral saturation was $88 \%$ on room air, heart rate $100 \mathrm{bpm}$, arterial pressure $110 / 70 \mathrm{mmHg}$. She was therefore admitted to the Respiratory Unit at the University L. Vanvitelli, Monaldi Hospital. Blood gas analysis showed acute hypocapnic respiratory failure (pH 7.45, $\mathrm{pCO}_{2} 32 \mathrm{mmHg}, \mathrm{pO}_{2} 59 \mathrm{mmHg}, \mathrm{HCO}_{3}-22.2 \mathrm{mmol} / \mathrm{L}$ ). Physical examination revealed inspiratory gasps partially modified by coughing in the lower right field, wheezing and inspiratory gasps in the upper left field, reduced vesicular murmur in the middle and lower left field. Chest X-ray showed only a left retrocardiac basal opacity whilst chest CT scan exhibited persistence of left lower lobe opacity, and a novel middle lobe parenchymal 
GGO with centrilobular tree in bud nodules and bronchiectasis; in both upper lobes ground glass opacities were reported. We started azithromycin, Vitamin C, N-acetylcysteine, prednisolone, aerosol therapy and enoxaparin 4000 IU once/day. Respiratory support through high flow nasal cannula oxygen therapy with $\mathrm{FiO}_{2} 25 \%$ Flow $25 \mathrm{~L} / \mathrm{min}$ was administered. Other tests were performed including QuantiFERON TB test, multiplex PCR nasopharyngeal swab for respiratory viruses, autoimmune profile and blood cultures. Blood examination revealed increase in CRP with normal white blood cell count; other tests were normal. The nasopharyngeal swab documented the presence of Coronavirus OC43. Daily chest monitoring was performed using lung ultrasound [10]. Clinical and physical examination improved after eight days of medical therapy; however high-resolution CT of the chest showed enlarged consolidation in left lower and middle lobe and more extensive ground glass areas in all lobes, overlapping of interlobar interstitial thickening, like crazy paving; no pleural effusion, no mediastinal or hilar lymph nodes were reported (Figure 1). Differential diagnosis, according to radiological pattern of CT scan diagnostic hypotheses were as follows: lung infection, alveolar proteinosis and lepidic heteroplastic growth. Multiple transbronchial biopsies, bronchoalveolar lavage, bronchoaspirate were subsequently performed. The left bronchial mucosa was hyperemic with profuse hematic suffusion from the basal segment. Tumor markers were also performed and strong positivity for CA 15-3 was recorded. At this stage a second Multiplex PCR nasopharyngeal swab for respiratory viruses confirmed the positive resolution of Coronavirus OC43 infection. The definitive histological diagnosis was micropapillary infiltrating adenocarcinoma, negative for EGFR, KRAS, BRAF, ROS1 receptor mutation, ALK translocation, with moderate PD-L1 expression (Tumor Proportion Score $1-49 \%$. Staging was completed with CT-PET scan. Before starting chemotherapy on the basis of the atypical radiological pattern, we performed both serological immune test and RT- PCR on nasopharyngeal swab for SARS-CoV2 virus, which resulted negative.

\section{Discussion}

The recent COVID-19 outbreak highlights the relevant role of viruses in prompting acute respiratory damage among lung cancer patients resulting in poor clinical outcomes. Several factors influence the enhanced risk of infection among lung cancer patients.
Firstly, the presence of central airway obstruction may favor atelectasis and/or distal pneumonia [11]. Secondly, the disruption of the innate and adaptive response induced by malignancy, malnutrition and active cancer treatment (chemotherapy, radiotherapy, immune checkpoint inhibitors) [12-17]. Thirdly, interaction site between viruses and host cell may offer further insights into the pathogenesis of viral infection among cancer patients [18-20]. Coronavirus OC43 spike protein binds to 9-O-acetylated sialic acids (9-O-Ac-Sias) attached as terminal residues to glycan chains on glycoproteins and lipids [21,22]. Sialic acids are constitutive components of cell surface glycoproteins and gangliosides and are frequently found in secreted glycoconjugates and in oligosaccharides, mainly of blood serum and mucus secretions [23]. Notably, the total sialic acid in serum or glycolipid-bound sialic acid are known to be elevated in different malignancies including lung cancer [24,25]. Furthermore, serum total sialic acid levels in lung cancer patients with metastatic disease were found higher than in patients with limited disease [25]. These findings suggest that coronavirus OC43-host cell interaction could play a relevant role both favoring viral infection and systemic spread among patients with lung cancer. In addition, viral pneumonia diagnosis in patients with lung adenocarcinoma is not straightforward. Symptom burden of both diseases - including fever, cough, dyspnea, asthenia may overlap potentially mimicking other respiratory diseases, finally resulting in late diagnosis [26]. Likewise, the CT hallmarks of $\beta$-coronaviruses (SARS, MERS, HKU-1) include mild or extensive GGOs, consolidations or mixed patterns with predominantly peripheral distribution [9]. In these cases, early differential diagnosis with cancer disease progression can be difficult to deduce without a multidisciplinary approach. In our patient, the rapid symptom deterioration coupled with laboratory findings prompted the diagnosis toward an acute viral infection. Finally, identification of viral pathogen on biological samples through acid nucleic amplification may significantly differ based on specimens [27]. The development of broadly reacting pan-coronavirus primers while allowing rapid detection of several coronavirus strains, may however result in limited sensitivity when compared to primers designed for each of the human strains $[28,29]$. This is essential for treatment decision making in coronavirus related-pneumonia. Despite the established absence of licensed therapy, recent evidences suggest that some drugs may exert potential antiviral activity including macrolides, immunoregulatory and antivirals agents [30-32]. In our case the treatment prompted from initial pathogen identification led to early intervention with azithromycin and high dose of

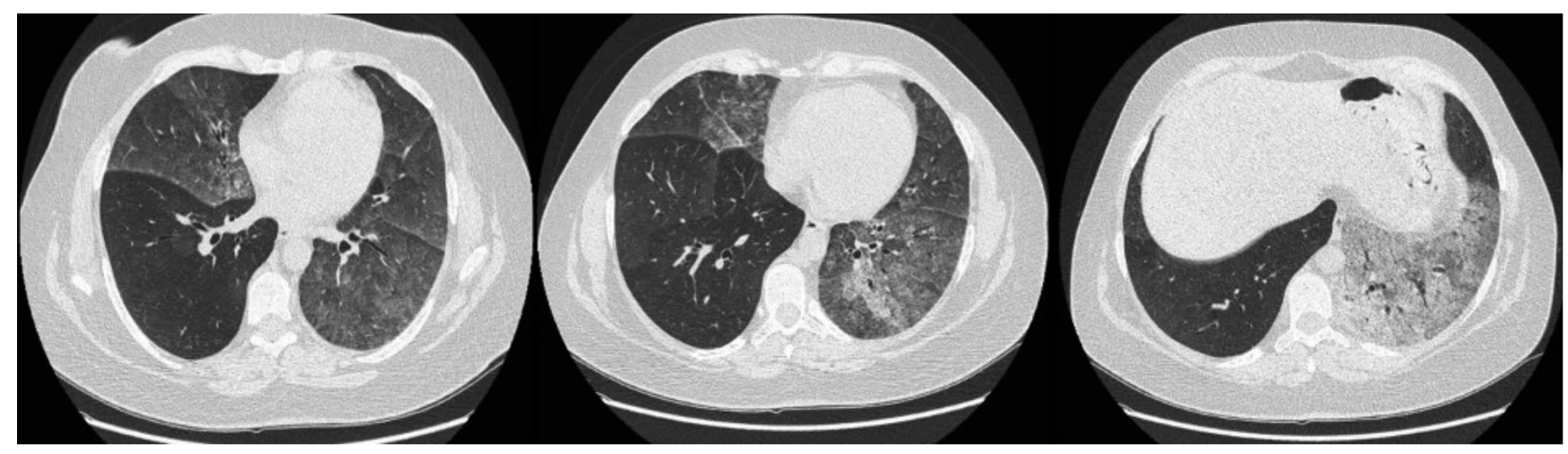

Figure 1. Lung CT scan showing left lower lobe adenocarcinoma with mixed consolidative and GGOs pattern; in the middle lobe CT showed the presence of GGOs along with interlobular thickening configuring a crazy paving pattern. 
antioxidants along with supportive high flow nasal cannula oxygen. In conclusion, our case demonstrates the relevance of coronavirus infection among patients with lung cancer and the challenges in defining adequate clinical management. recent outbreak of the novel coronavirus SARS-CoV-2 highlights the urgency of further studies in lung cancer patients.

\section{References}

1. Passaro A, Peters S, Mok TSK, et al. Testing for COVID-19 in lung cancer patients. Ann Oncol 2020;31:832-4. doi: 10.1016/j.annonc.2020.04.002.

2. Perrotta F, Matera MG, Cazzola M, Bianco A. Severe respiratory SARS-CoV2 infection: Does ACE2 receptor matter? Respir Med 2020;68:105996.

3. Boccia M, Aronne L, Celia B, et al. COVID-19 and coagulative axis: review of emerging aspects in a novel disease. Monaldi Arch Chest Dis 2020;90:1300. doi: 10.4081/monaldi. 2020.1300 .

4. Perrotta F, Corbi G, Mazzeo G, et al. COVID-19 and the elderly : insights into pathogenesis and clinical decision-making. Aging Clin Exp Res 2020. doi:10.1007/s40520-020-01631-y.

5. Witt C. European respiratory society/american thoracic society/international association for the study of lung cancer international multidisciplinary classification of lung adenocarcinoma: state of the art. J Thorac Oncol 2011;6:1451.

6. Cohen JG, Reymond E, Jankowski A, et al. Lung adenocarcinomas: correlation of computed tomography and pathology findings. Diagn Interv Imaging 2016;97:955-63.

7. West HJ. Managing multifocal bronchioloalveolar carcinoma/ lepidic predominant adenocarcinoma: changing rules for an evolving clinical entity. Clin Adv Hematol Oncol 2014;12: 593-600.

8. Gardiner N, Jogai S, Wallis A. The revised lung adenocarcinoma classification-an imaging guide. J Thorac Dis 2014;6: S537-46.

9. Koo HJ, Lim S, Choe J, et al. Radiographic and CT features of viral pneumonia. Radiographics 2018;38:719-39.

10. Rinaldi L, Milione S, Fascione MC, et al. Relevance of lung ultrasound in the diagnostic algorithm of respiratory diseases in a real-life setting: A multicentre prospective study. Respirology 2019;25:535-42. doi:10.1111/resp.13659.

11. Fiorelli A, Perrotta F, Mollica M, et al. Endoscopic central airway recanalization to enable first line pembrolizumab treatment in a PD-L1 strongly positive non-small cell lung cancer: a case report. J Cardiothorac Surg 2019;14:50.

12. Bianco A, Malapelle U, Rocco D, et al. Targeting immune checkpoints in non small cell lung cancer. Curr Opin Pharmacol 2018;40:46-50.

13. Perrotta F, Rocco D, Vitiello F, et al. Immune checkpoint blockade for advanced NSCLC: A new landscape for elderly patients. Int J Mol Sci 2019;20. doi:10.3390/ijms20092258.

14. de Blasio F, Di Gregorio A, de Blasio F, et al. Malnutrition and sarcopenia assessment in patients with chronic obstructive pulmonary disease according to international diagnostic criteria, and evaluation of raw BIA variables. Respir Med 2018;134:1-5.

15. Di Zazzo E, Polito R, Bartollino S, et al. Adiponectin as link factor between adipose tissue and cancer. Int $\mathrm{J}$ Mol Sci 2019;20. doi:10.3390/ijms20040839.

16. de Blasio F, de Blasio F, Miracco Berlingieri G, et al. Evaluation of body composition in COPD patients using multifrequency bioelectrical impedance analysis. Int $\mathrm{J}$ Chron Obstruct Pulmon Dis 2016;11:2419-26.

17. Perrotta F, Nankivell M, Adizie B, et al. Endobronchial ultrasound-guided transbronchial needle aspiration for PD-L1 testing in non-small cell lung cancer. Chest 2020. doi: 10.1016/j.chest.2020.04.059.

18. Cattaneo F, Guerra G, Parisi M, et al. Expression of formylpeptide receptors in human lung carcinoma. Anticancer Res 2015;35:2769-74.

19. Nigro E, Imperlini E, Scudiero O, et al. Differentially expressed and activated proteins associated with non small cell lung cancer tissues. Respir Res 2015;16:74.

20. Nigro E, Stiuso P, Matera MG, et al. The anti-proliferative effects of adiponectin on human lung adenocarcinoma A549cells and oxidative stress involvement. Pulm Pharmacol Ther 2019;55:25-30.

21. Krempl C, Schultze B, Herrler G. Analysis of cellular receptors for human coronavirus OC43. Adv Exp Med Biol 1995;380:371-4.

22. Walls AC, Park Y-J, Tortorici MA, et al. Structure, function, and antigenicity of the SARS-CoV-2 spike glycoprotein. Cell 2020;181:281-92.e6.

23. Schauer R, Srinivasan GV, Wipfler D, et al. O-Acetylated sialic acids and their role in immune defense. Adv Exp Med Biol 2011;705:525-48.

24. Zhou X, Yang G, Guan F. Biological functions and analytical strategies of sialic acids in tumor. Cells 2020;9. doi:10.3390/cells9020273.

25. Suer Gokmen S, Kazezoglu C, Tabakoglu E, et al. Serum total sialic acid levels in lung cancer patients of different histological types with and no extrapulmonary metastases. Turk J Biochem 2004;29:262-7.

26. Maniscalco M, Bianco A, Mazzarella G, Motta A. Recent advances on nitric oxide in the upper airways. Curr Med Chem 2016;23:2736-45

27. Wang $\mathrm{W}, \mathrm{Xu}$ Y, Gao R, et al. Detection of SARS-CoV-2 in different types of clinical specimens. JAMA 2020;323:1843-4.

28. Gerna G, Campanini G, Rovida F, et al. Genetic variability of human coronavirus OC43-, 229E-, and NL63-like strains and their association with lower respiratory tract infections of hospitalized infants and immunocompromised patients. J Med Virol 2006;78:938-49.

29. Gaunt ER, Hardie A, Claas ECJ, et al. Epidemiology and clinical presentations of the four human coronaviruses 229E, HKU1, NL63, and OC43 detected over 3 years using a novel multiplex real-time PCR method. J Clin Microbiol 2010; 48:2940-7.

30. Barlow A, Landolf KM, Barlow B, et al. Review of emerging pharmacotherapy for the treatment of coronavirus disease 2019. Pharmacotherapy 2020;40:416-37.

31. Grein J, Ohmagari N, Shin D, et al. Compassionate use of remdesivir for patients with severe Covid-19. N Engl J Med 2020;382:2327-36.

32. Gupta N, Agrawal S, Ish P. Chloroquine in COVID-19: the evidence. Monaldi Arch Chest Dis 2020;90:1290. doi:10.4081/ monaldi.2020.1290. 